\title{
Image Processing and Analysis at IPAG
}

\section{INTRODUCTION}

Medical image analysis has grown and evolved tremendously in the last 30 years. The distinctive nature of the problems encountered have led to the development of a significant body of work addressing such issues as fully three-dimensional data, nonrigid models for motion, deformation and comparison, and the statistical variation of normal and abnormal structure. This area of research derives from the clinical and scientific applications which must be well understood. However, the methodologies developed encompass an array of techniques that have advanced image analysis independent of the application.

This paper will describe the development of the Image Processing and Analysis Group (IPAG) at Yale and the broad range of work in the area of medical image analysis that has been pursued. Throughout our work, we have endeavored to take advantage of all available information both from prior knowledge of physical properties, geometric constraints or statistical variation as well as imaging data from various modalities. Medical imaging problems typically lie in a well-defined domain. The challenge is to take best advantage of the given domain to solve the often complex and subtle problems posed.

\section{ORIGIN AND HISTORY}

\section{0's to 1983: Initial Ph.D. Faculty in Diagnostic Radiology}

During the 1970's, several Ph.D. faculty were hired into the Department of Diagnostic Radiology in the medical school at Yale with their primary tasks being aimed at providing physics support for the clinical operations in the burgeoning fields of nuclear medicine (Bob Lange), ultrasound (Fred Kremkau) and computed tomography (Stelios Orphanoudakis). Each of these faculty members were able to initiate and maintain their own research programs as well, using different methods to support their efforts, including industrial and some federal funding. In 1982, as Magnetic Resonance was just beginning to be considered for clinical use, John Gore was hired as the NMR physicist, now with a clear charge to develop a research program in this area. Soon after (1982-1983), three more hires were made within the Department of Diagnostic Radiology. Art Gmitro and Gene Gindi were hired initially to help with image/signal processing and hardware issues related to the development of a Digital Subtraction Angiography (DSA) system, funded by an industrial grant and Jim Duncan was hired jointly by the Department and the Section of Cardiology to work on a number of image analysis issues related to the TIMI (Thrombolysis in Myocardial Infarction) project funded by the National Institutes of Health. While all of these faculty were hired for separate reasons, this group began to get together and develop a number of interactions to start to form a medical imaging methodologybased research community at Yale.

As the social and professional relationships among this group of seven or so faculty members matured, these people formed, with support from clinical colleagues such as Carl Jaffe, Richard Greenspan and Barry Zaret, the substrate for the bioimaging science research now firmly established at Yale in 2003.

While there were a variety of attempts to integrate bioimaging science into a single entity within Diagnostic Radiology, by and large these research efforts clustered into three primary areas: nuclear medicine, magnetic resonance imaging and image post-processing. In this paper, we will focus on the evolution of the latter area, that became the Yale Image Processing and Analysis Group (IPAG). However, at the end of the paper, we will describe how the early possibilities of an integrated bioimaging science community were re-kindled, bringing the evolution of effort full circle.

\section{3-1989: Biomedical Image Processing Early Years}

One subset of the Ph.D. researchers in the bioimaging sciences areas overlapped significantly enough to form a core effort in medical image processing: Drs. Duncan, Gindi, Gmitro and Orphanoudakis. Stelios Orphanoudakis obtained a joint appointment with the Department of Electrical Engineering (EE) within the Faculty of Arts and Sciences at Yale in the late 1970's. Jim Duncan and Gene Gindi also received joint appointments in Electrical Engineering. These faculty established new courses in the EE department in both Digital Image Processing and Computer Vision. While both had a biomedical flavor in the examples given, the courses were established in order to interest Yale graduate and undergraduate students in the general areas of image processing and image analysis. At the graduate level, this effort soon paid off. Several new graduate students with focused interests in image processing and analysis (Larry Staib, Kathy Andriole, Dimitris Gerogiannis and Volker Tresp) joined Scott Holland (already working with Stelios Orphanoudakis on ultrasound signal processing) as the core students of this new group. Early work on parallel image processing algorithms [1] and vessel tracking [2] appeared during this period. Other work by Art Gmitro and Gene Gindi focused on optical image processing strategies for feature extraction garnering a best paper award from the journal Optical Engineering [3]. In addition, a body of work on image reconstruction methods began at this time ranging from optical methods [4] to statistical methods in the work of Mindy Lee [5], [6] in collaboration with Gene Gindi and George Zubal, nuclear medicine physicist. This work also included the development of the Zubal Phantom [7], [8], a high-resolution three-dimensional anthropomorphic image phantom derived from segmented human anatomy of the full body representing all of the major structures. This data was designed for use as a phantom in imaging simulations such as for Monte Carlo methods.

The emphasis, however, from the outset was to bridge areas of general image processing and computer vision with medicalimaging-specific knowledge. As the Yale group was forming, much of the efforts in the medical image analysis field seemed to be coming from one of two directions: i.) computer vision and image processing specialists with an interest in, but limited detailed knowledge of, medical imaging and its applications 


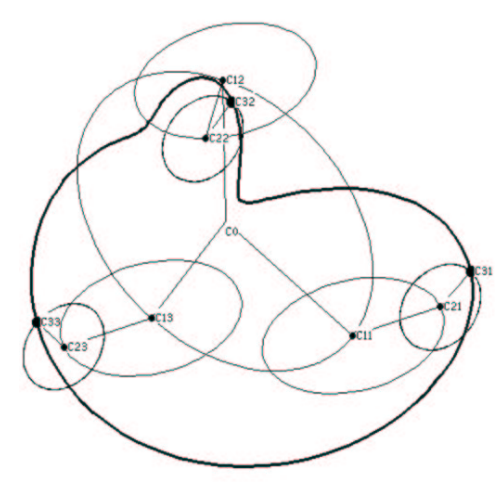

Fig. 1. In 2D, the elliptic Fourier parameters define a curve and, by way of probability distributions, can be used to constrain the boundary finding process.

or ii.) medical imaging physicists who understood the principles of image acquisition and formation, but who typically did not have the specific applied mathematics, computer science or signal and image processing background to contribute in the algorithm development areas. By being physically located in the medical school while maintaining close ties to engineering through teaching and research, we strove to advance the field by developing new image processing and analysis methodology fully grounded with knowledge of image acquisition physics, anatomy and physiology, and the clinical and scientific questions.

From about 1985 to 1988 there was a strong influence of work from artificial intelligence in computer vision and medical image analysis. Drs. Gindi and Duncan developed a course project in their computer vision course in which students implemented a "blocks world" image understanding system taking the problem from image acquisition to high level model matching [9]. Jim Duncan incorporated explicit knowledgebased concepts modeling the walls of the left ventricle of the heart in early work [10]. Advances in the application of neural networks to computer vision and medical image analysis were also made at this time in the group by Gene Gindi with his graduate student Joachim Utans [11]. They developed approaches to object recognition using hierarchical matching networks.

\section{9-present: IPAG Flourishes}

As 1990 approached, the group began to explore more deeply some of the key areas in medical image analysis. Larry Staib, Amir Amini and Hemant Tagare became faculty members in the group around this time creating a critical mass of image analysis researchers. Workstation computational and graphical power was also increasing tremendously enabling an array of techniques that were impossible before. The development of the web started in this period and we created one of the first web sites in medical image analysis in December 1993 (http://noodle.med.yale.edu/). The web has obviously since become an essential aspect of dissemination and research.

Below we will discuss some of the key areas that IPAG has pursued including deformable models for segmentation, nonrigid motion analysis aimed primarily at cardiac motion, image registration and structural measurement.
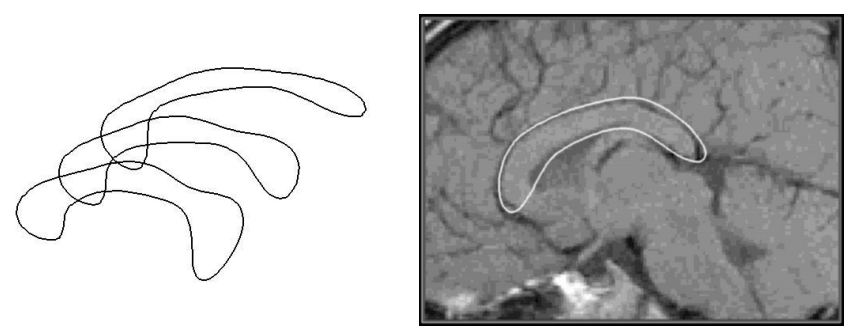

Fig. 2. On the left, a mean curve is shown in the center along with curves representing plus and minus one standard deviation of the model parameters. On the right this model is used to segment the corpus callosum from a magnetic resonance image.
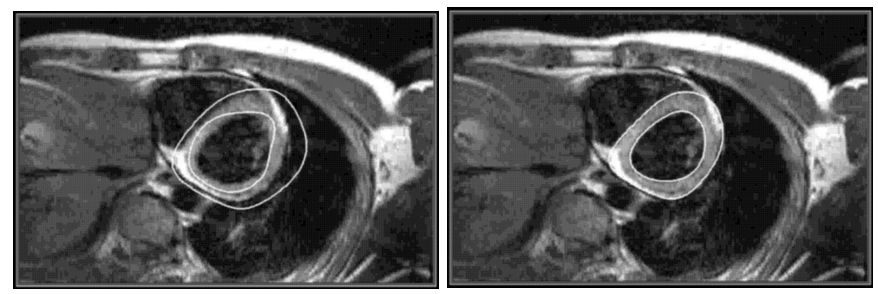

Fig. 3. 2D deformable Fourier model segmenting the myocardium from magnetic resonance images (left: initialization; right: final curves).

Deformable Models: A strong focus of the group arose at this time aimed at developing mathematical strategies for extracting quantitative measurements from medical images. Boundary finding for anatomical structure is a central problem that pervades many measurement problems. A chapter on the methodologies discussed below was included in a collection on medical imaging [12]. The key themes that emerged from this work were the incorporation of prior information of shape as constraints or biases and the integration of multiple information sources [13], [14]. Such information is crucial to the solution of medical image segmentation problems in the face of noise, ambiguity and structural complexity. We approached such segmentation problems from the perspective of mathematical optimization and (Bayesian) estimation theory, solved using a variety of numerical approaches. Perhaps the earliest clear example of this trend was the Ph.D. thesis work of Larry Staib [15]. This work was aimed at developing a concise parametrized model, using Fourier descriptors, of a contour or surface that could then be deformed or modified to find a specific boundary within an image [16], [17], [18]. This model easily allows the incorporation of prior shape information and was the first technique to use prior shape information in a general and flexible way.

A maximum a posteriori objective function of parameters, $\mathbf{p}$, was used and allows a trade-off or compromise between prior information, $\operatorname{Pr}(\mathbf{p})$, and image-derived information, $\operatorname{Pr}(b \mid \mathbf{p})$. For a uniform prior, this formulation reduces to the maximum likelihood solution.

$$
\mathbf{p}_{\text {map }}=\arg \max _{\mathbf{p}}[\ln \operatorname{Pr}(\mathbf{p})+\ln \operatorname{Pr}(b \mid \mathbf{p})]
$$

Prior probability distributions on the parameters are used to introduce a global shape model with a bias toward an expected range of shapes. This approach is illustrated in Figures 1, 2, 3 and 4. This work was initially presented in 1988 [19], [20]. 

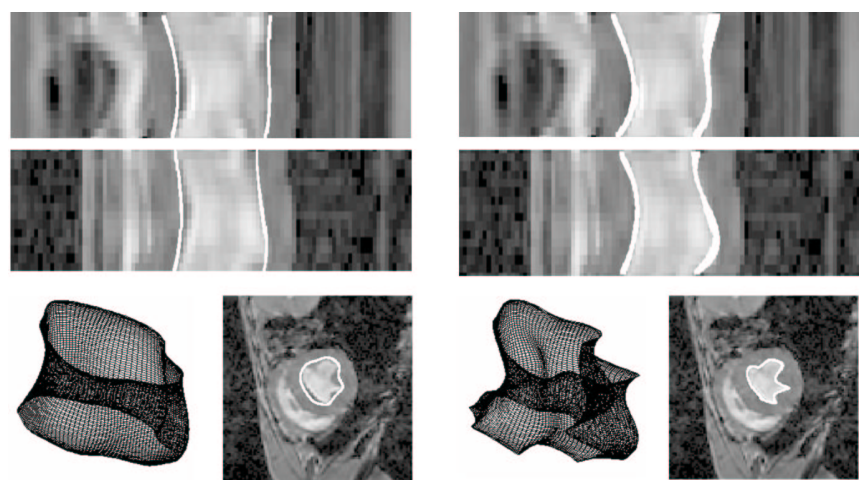

Fig. 4. Spatiotemporal models can be used to solve boundary finding problems in temporal sequences as in this cardiac left ventricle magnetic resonance example showing three orthogonal views with the initialization of the left and the final result on the right.
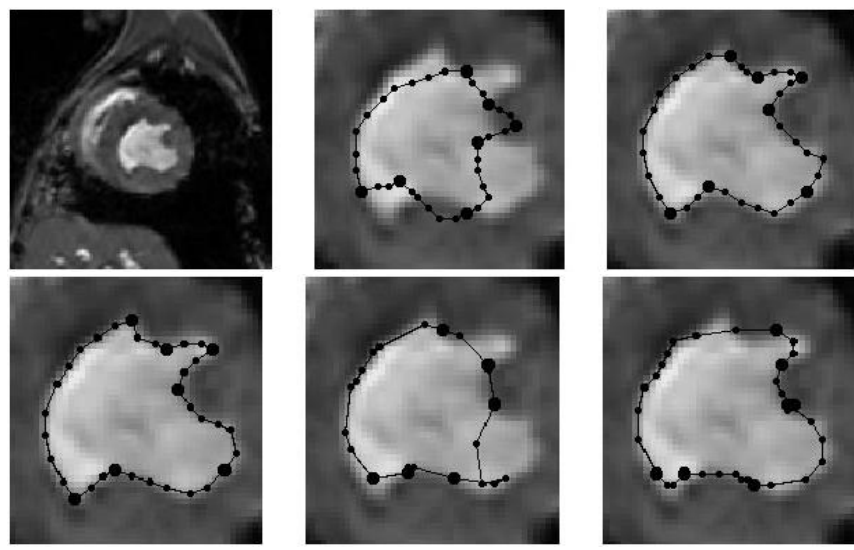

Fig. 5. Original MR cardiac image (top left) with mean contour (top middle) and expert tracing (bottom left). Note the improvement in performance moving from an independent covariance (bottom middle) to smoothness covariance (bottom right) and finally the prior shape covariance (top right).

Interestingly from a historical perspective, this was being developed in parallel with the deformable "snake" boundary finding approaches of Kass, Witkin, and Terzopoulos.

Later, we studied active shape models [21] and showed the relationship to Fourier models and ways of exploiting the use of the covariance matrix [22], [23] (see Figure 5).

Two alternate approaches to using prior knowledge were pursued by Hemant Tagare. When the prior probability distributions of the boundaries are unknown, an alternate approach is available which uses only a single canonical shape for the boundary. The canonical shape is encoded in a template which fits the image boundary by deforming to minimize an energy function. Using conformal maps, normals to a deformable 2$\mathrm{d}$ template can be extended smoothly to a large region of space [24]. The template is deformed by moving it along the extended normals - which are called "orthogonal curves". Interestingly, it is rather straightforward to show that the orthogonal curves and dynamic programming gives the global minimum of the energy function. Finding the global minimum is crucial to avoiding local minima that arise from noise and give grossly incorrect answers. This algorithm has been successfully used for many years to segment carpal bones in CT scans. Figure 6 shows an example.

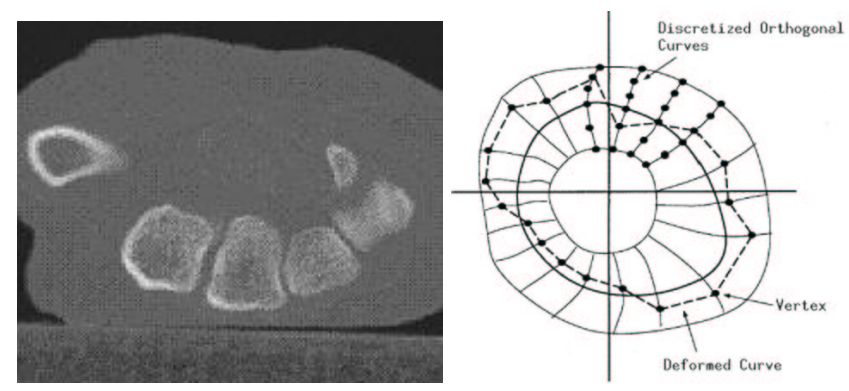

Fig. 6. A wrist CT image showing carpal bones (left) and a deformable template with orthogonal curves and an example deformation (right).

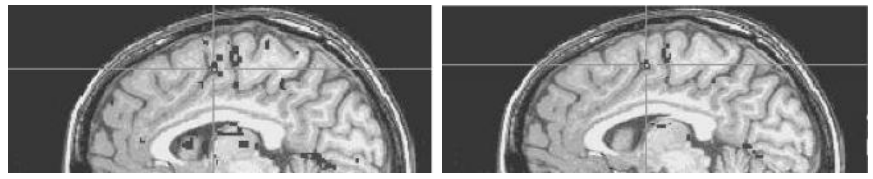

Fig. 7. Functional activation map with global registration (left) and with local registration (right) determined using the level set algorithm with shape priors to segment and register the corpus callosum.

In the second approach, prior knowledge about the shape of the boundaries was incorporated into a level set formulation of active contours in a variational framework. The shape of a boundary is all of the information that is left when location, orientation, and size information is removed. The shape prior is mapped into the image space by a translation, rotation and scaling and it appears as an additional term in the energy function of the level set [25]. During level set evolution, the translation, rotation and scaling are continuously estimated and updated. The algorithm simultaneously achieves segmentation of an image and its registration with a standard coordinate system. This method can be exploited in fMRI studies where simultaneous segmentation and registration of local brain structures leads to a lowering of false positives in the activation map as shown in Figure 7.

The results of active contours are often evaluated by comparison with manual segmentation. Unfortunately, experimental evaluations often do not shed light on when and why active contours become inaccurate. A theoretical analysis is required for understanding this. In his Ph.D. thesis, Tianyun Ma theoretically explored accuracy and consistency in active contours [26], [27]. He was able to show that some of the common forms of external energy functions can lead to a significant bias in segmentation. Some energy functions can also make the contour unstable. By suitable modifications of the energy function, the bias and stability problems can be eliminated [26], [27].

Integration: Boundary finding based on edge features can be augmented by considering the incorporation of region-based information and developing an integrated Maximum a posteriori (MAP) probability method in order to determine the surface (or curve, in 2D) parameters which correspond to the structure which matches both the boundary strength in the image and the region homogeneity properties [13], [28]. This work on integration was the thesis work of Amit Chakraborty.

We consider two modules: one related to boundary finding and the other region growing where each contains a coupling 


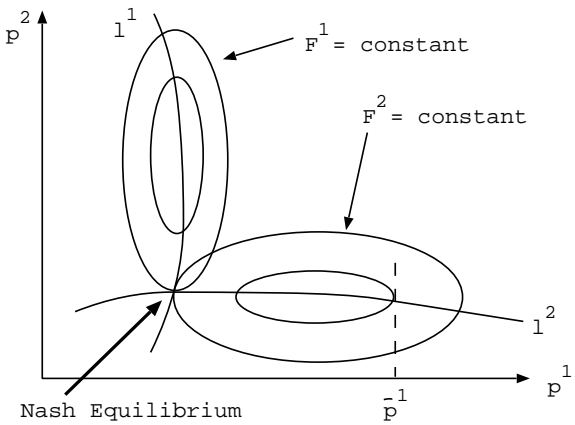

Fig. 8. In a simple 2D game theoretic formulation, constant level curves for $F^{1}(\cdot)$ and $F^{2}(\cdot)$ and the corresponding reaction curves $\left(l^{1}\right.$ and $\left.l^{2}\right)$ of the two players are shown. For a fixed $p^{1}=\bar{p}^{1}$, the best player 2 can do is to minimize $F^{2}$ along the line $p^{1}=\bar{p}^{1}$. For each different $\bar{p}^{1}$, a different optimal response can be found for player 2 , and the collection of all these points form $l^{2}$, the reaction curve of player 2 . The Nash equilibrium lies at the intersection of the players reaction curves.

term that feeds information related to the other module:

$$
\begin{aligned}
\min _{X} F^{1}(X, p) & =\min _{X}\left[f_{1}(X)+\alpha f_{21}(X, p)\right] \\
\min _{p} F^{2}(p, X) & =\min _{p}\left[f_{2}(p)+\beta f_{12}(p, X)\right]
\end{aligned}
$$

$X$ represents the output of the region-based process and $p$ represents the output of the boundary finding module. In the first equation, $f_{1}(X)$ represents region-based classification information [13]. In the second, $f_{2}(p)$ represents the boundary strength in the shape prior-driven contribution. $f_{12}(p, X)$ and $f_{21}(X, p)$ represent interaction terms between the two modules. Initially, these equations were solved sequentially with $\alpha=$ 0 . The region based segmentation was determined first and then that information was used to optimize the boundary.

A more powerful approach to integration comes from a game theoretic formulation [29], [30]. We were able to derive a more general formulation pioneering the use of game theory (started by the thesis work of Iș1l Bozma [31]) as a means for integrating image information [14].

When $\alpha$ is non-zero, the first equation includes an additional interaction term, $f_{21}(X, p)$, which feeds back the latest available output $p$ of the boundary module and represents the agreement of the voxels within the current boundary with the assumed gray level distribution for the indicated tissue type. In the second equation, the interaction term $f_{12}(p, X)$ uses the latest available output $X$ of the region process. The modules assume the roles of players in a 2-player game and are optimized in parallel. The game continues until the players can not improve their positions without cooperation from the other player. This natural stopping point of the parallel decision making process constitutes the Nash equilibrium solution [32] (see Figure 8).

The rational decision provided by the Nash equilibrium solution is the natural counterpart of the optimum found with sequential objective optimization. We have found it to be more robust to noise and initialization in a variety of $2 \mathrm{D}$ and $3 \mathrm{D}$ problems [13].

In order to compute the contribution of the region based information, we need to compute an integral over the region. We can compute this efficiently if we convert the volume integral

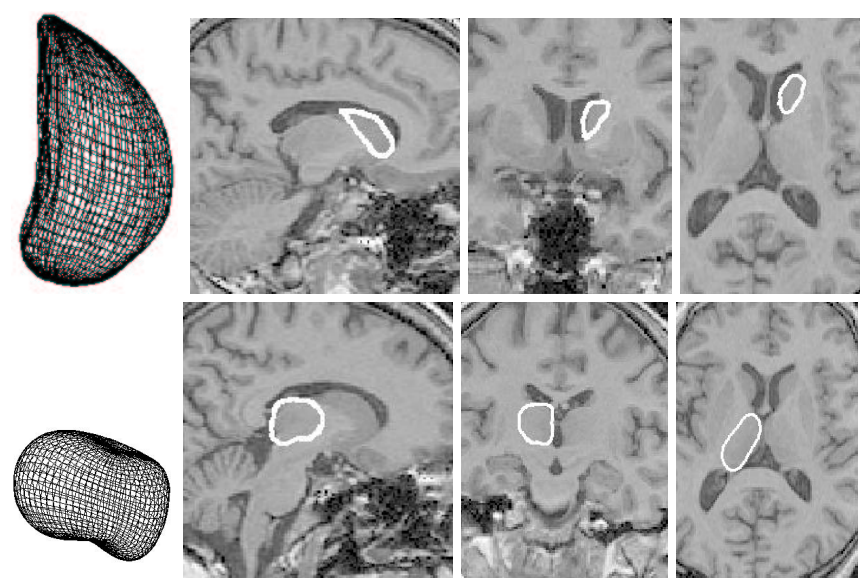

Fig. 9. Results of surface finding for the head of the left caudate nucleus (top row) and the right thalamus (bottom row) in an MR image. The wireframe and three perpendicular slices through the $3 \mathrm{D}$ image $\left(1.2 \mathrm{~mm}^{3}\right.$ voxels $)$ are shown with the surface obtained using both boundary and region information.

to an area integral using Gauss' divergence theorem [33]. We construct a function whose divergence is the function we wish to integrate by integrating in each of the coordinate directions. Then we can simply compute the area integral of this function during the optimization process (see [28] for details), greatly reducing the necessary computation.

In the brain, some subcortical structures often have poor contrast between gray and white matter because they are striate and appear with intermediate intensity. These structures, however, are less variable, in terms of shape, than the cortex. Thus, prior shape information can be of great value in identifying subcortical boundaries. In Figure 9, we demonstrate the performance of the 3D integrated method on subcortical structure examples: the head of the right caudate nucleus and the left thalamus. Using the integrated method with a prior shape model, along with region and boundary information, the proper boundaries are found.

Level Set Method Incorporating Thickness Constraint: While some segmentation problems are well suited to the constraints that global shape information provides, some involve structures whose shapes are highly variable or have no consistent shape at all and thus require more generic constraints [12].

Xiaolan Zeng, in her graduate work in the group, developed a coupled surfaces approach for automatically segmenting a volumetric layer from a 3D image [34], [35], [36]. This approach uses a set of coupled differential equations, with each equation determining the evolution or propagation of a surface within a level set framework. In the case of the cortex, one surface attempts to localize the white matter/gray matter (WM/GM) inner cortical boundary and the other the gray matter/cerebrospinal fluid (GM/CSF) outer boundary. Coupling between the surfaces incorporates the notion of an approximately fixed thickness separating the surfaces everywhere in the cortex. This soft constraint helps in ensuring that the GM/CSF boundary is captured even in the deeper cortical folds in the brain. A further assumption is that across each surface, there is a local difference in the gray level values, while in between the two surfaces there is a homogeneity of gray levels. By evolving two embedded surfaces simultaneously, each driven by its own image-based infor- 


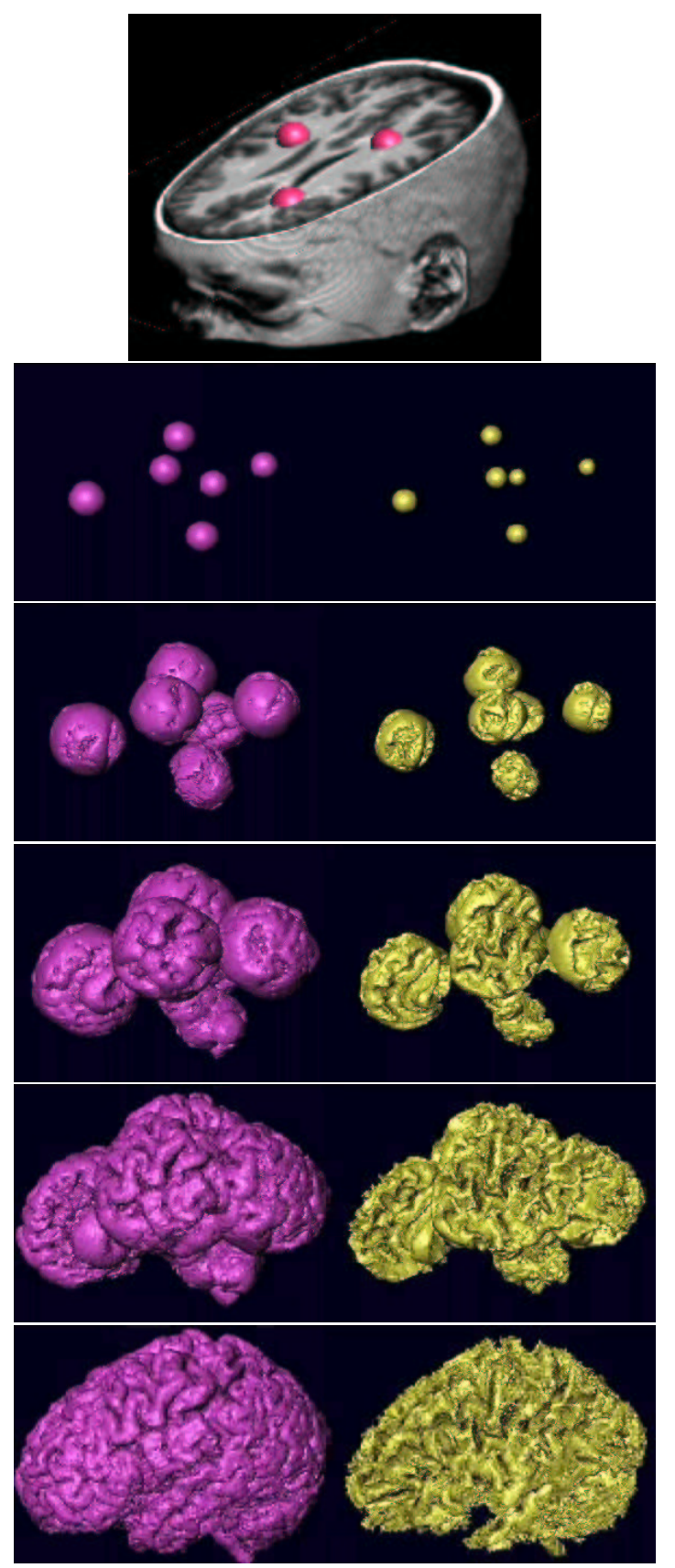

Fig. 10. Evolution of coupled level sets: outer (magenta) and inner (yellow) surfaces propagate from initialized pairs of concentric spheres in a 3D MR brain image to localize the gray matter.

mation while maintaining the coupling, we are able to achieve an automatic and robust segmentation of the cortex, and simultaneously obtain a representation of the inner and outer cortical surfaces.

Starting from inside the inner bounding surface (gray/white boundary), with an offset in between, the interfaces propagate along the normal direction stopping at the desired place, while maintaining the distance between them. Embedding each surface as the zero level set in its own level function, we have two equations: $\partial \Psi_{i n} / \partial t+F_{i n}\left|\nabla \Psi_{i n}\right|=0$ and $\partial \Psi_{\text {out }} / \partial t+F_{\text {out }}\left|\nabla \Psi_{\text {out }}\right|=0$ where $F_{\text {in }}$ and $F_{\text {out }}$ are functions of the surface normal direction, image-derived informa-

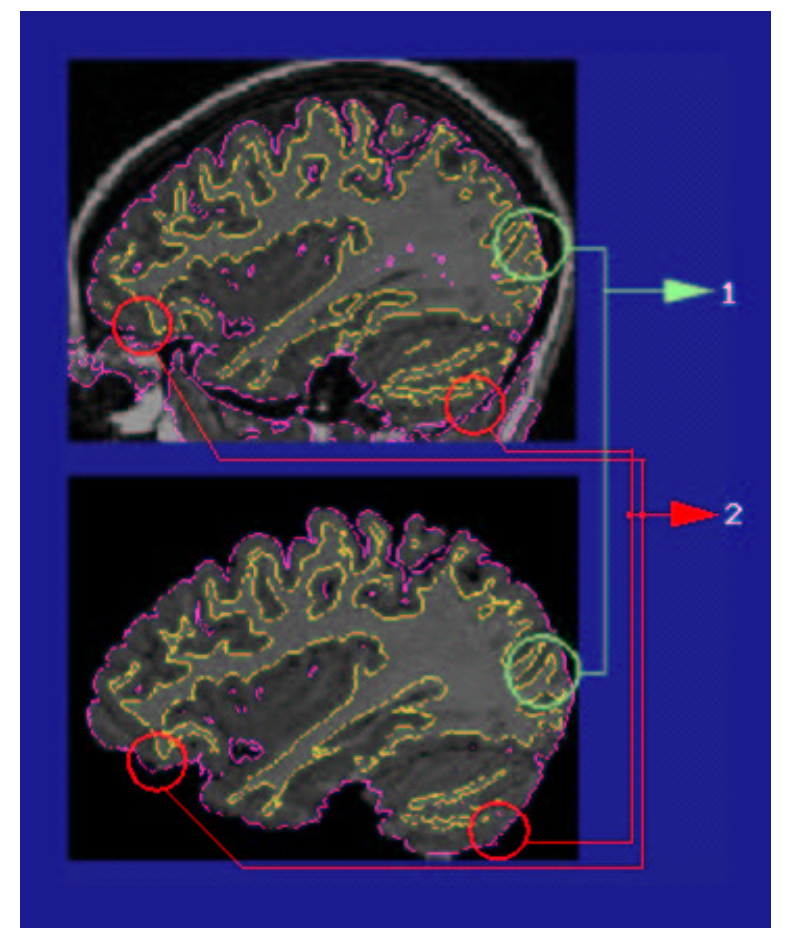

Fig. 11. Top: Surfaces resulting from single surface approach finding the inner and outer cortical surfaces separately; Bottom: Coupled surfaces approach run on same data overlaid on a sagittal slice masked by expert tracing of the outer cortical surface showing good agreement. Coupling prevents the inner surface from collapsing into CSF (1) and the outer surface from penetrating non-brain tissue (2).

tion and the distance between the two surfaces. The coupling is embedded in the design of $F_{\text {in }}$ and $F_{\text {out }}$. Where the distance between the two surfaces is within the normal range for cortical thickness, the two surfaces propagate according to the image-based information; where the distance between the two surfaces is out of the normal range, the distance constrains the propagation. We define:

$$
\begin{aligned}
F_{\text {in }} & =g\left(p_{G M-W M}\left(\vec{\theta}^{*}\right)\right) h\left(\Psi_{\text {out }}\right) \\
F_{\text {out }} & =g\left(p_{C S F-G M}\left(\vec{\theta}^{*}\right)\right) h\left(\Psi_{\text {in }}\right) .
\end{aligned}
$$

Function $g$ smoothly maps larger gray level transition probability to slower speed. Function $h$ smoothly penalizes the distance outside of the normal range. Thus, each surface moves with constant speed along the normal direction, and slows down or stops when either the image-based information becomes strong or the distance to the other surface moves away from the normal range.

The ability of the evolving level set to change topology, break, merge and form sharp corners greatly assists in the convergence to complex structures. In addition, the level set approach facilitates structural measurement through the direct computation of geometric parameters, such as curvature and thickness, via the level set function [36] (see, for example, Figure 18). Surface renderings showing the evolution of the inner and outer cortical layers found from a normal 3D brain MR image using this approach are shown in Figure 10. This method has been extensively tested in our lab and elsewhere [37] with accurate results under a range of conditions. 


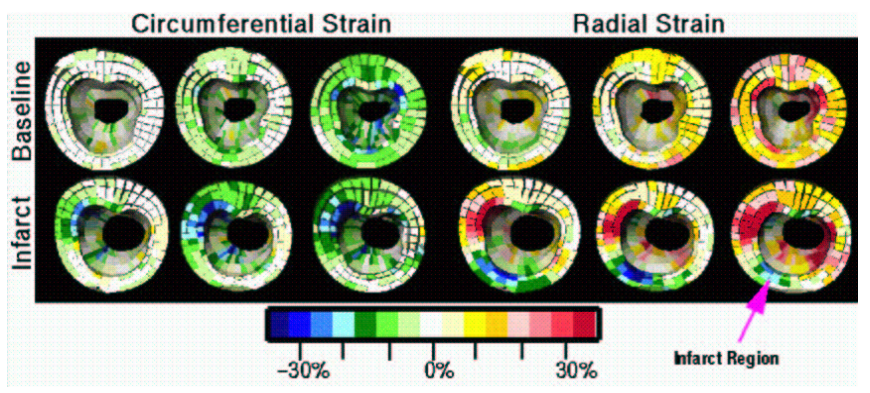

Fig. 12. Strain computed using a biophysical model from canine cardiac MR data comparing baseline to infarcted left ventricle.
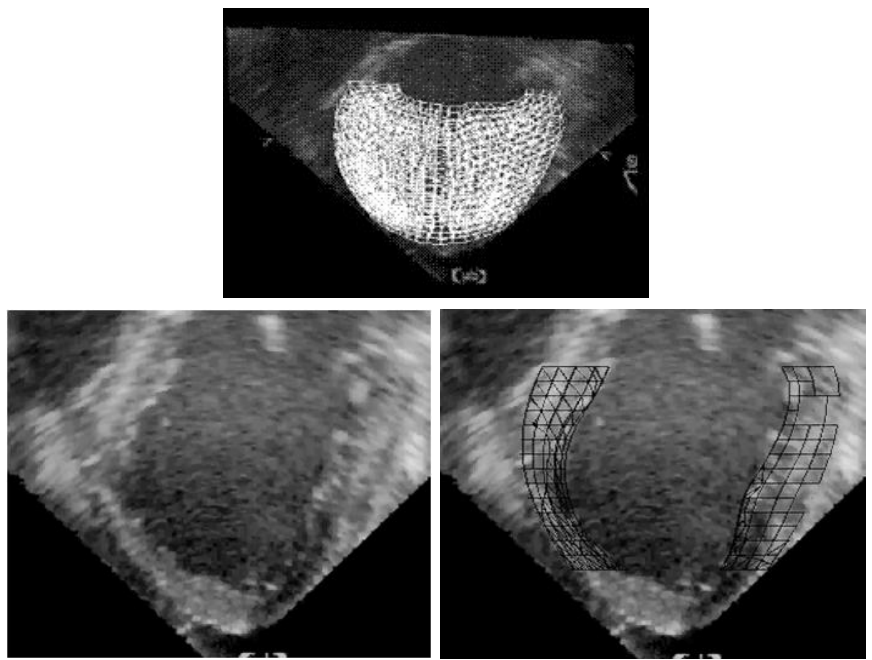

Fig. 13. Echo ultrasound data can also be analyzed with a physical model to estimate strain. Shown here are points derived from the model overlaid on the ultrasound image to give "echo tissue tags" analogous to MR tagging.

Nonrigid Motion Leading to Deformation: Analyzing information embedded in temporal sequences of images had long been of interest to medical image analysis researchers, with many of the driving applications coming from the need to quantify cardiovascular function. The IPAG efforts focused primarily on developing strategies to follow left ventricular (LV) motion, and ultimately deformation, in attempts to stratify differences in LV performance as derived from any one of several non-invasive imaging modalities. A comprehensive review of methods for estimation of cardiac motion and deformation including the methods described below was included in a recent book on medical image analysis [38].

In the early 1990's, a number of groups had begun to develop techniques to find correspondences between pairs of frames from 2D cardiac image sequences [39], [40] as well as to develop useful parametrizations for spatiotemporal datasets [41], [42] in attempts to model and more fully determine nonrigid motion. In the computer vision community, these efforts were initially reported at the first conference session on nonrigid motion analysis at the IEEE Computer Society's 1991 Conference on Computer Vision and Pattern Recognition (CVPR91). To the clinical cardiology community, however, many of these ideas were seen as the most recent attempts to address the difficult problem of stratifying left ventricular function.

Within IPAG, our focus was to develop a strategy for quanti- fying LV function that could be used to derive information from any one of a number of noninvasive image sequences. While other groups focused almost solely on the promising techniques of analyzing MR tagging [43], [44] or MR phase velocity [45], we chose to try to develop approaches that were potentially modality-independent. The common thread we saw was that if we could somehow accurately segment out the endocardial and epicardial boundaries from each frame of a cardiac image sequence, and sample often enough, we could assume that local surface shape would be preserved between any two frames and hence be used as a tracking metric.

This effort began by analyzing two-dimensional cardiac image sequences. Due to its spatial resolution, and in part to be able to compare to tagging and phase velocity tracking results, we chose to use two-dimensional cine-gradient echo MR image sequences as our initial data. Endocardial and epicardial boundary segmentation in each frame was performed using modified versions of the Bayesian boundary finding strategy described above (see [16]). Curvatures, $\kappa$, were derived at each boundary point and used as tracking tokens. Matching was performed by finding the best local segment of length $L$ surrounding point $p_{i+1}$ at frame i+1 within a plausible search region $W$ whose shape best matches a segment surrounding candidate point $p_{i}$ at time i via a squared curvature (i.e. bending energy) metric:

$$
\hat{p}_{i+1}=\arg \min _{p_{i+1} \in W} \sum_{l=1}^{L}\left(\kappa_{l}\left(p_{i+1}\right)-\kappa_{l}\left(p_{i}\right)\right)^{2}
$$

Once the optimal $p_{i+1}$ is found in the search region, the vector $u$ that connects these two points can be defined. Similar displacement vectors can be located at all points $p_{i}$ with confidences, $C$, assigned by the strength and uniqueness of the shape match. Uncertainty, noise and out-of-plane motion were handled by employing a regularization strategy to estimate a final set of smoothed displacements:

$\hat{\mathbf{u}}(p)=\arg \min _{\overrightarrow{\mathbf{u}}} \int_{P} \int_{V}\left[C_{\overrightarrow{\mathbf{u}}}(p)\left\{\overrightarrow{\mathbf{u}}(p)-\overrightarrow{\mathbf{u}}_{0}(p)\right\}^{2}+\frac{\partial \overrightarrow{\mathbf{u}}(p)}{\partial \mathbf{p}}\right] d \mathbf{p}$

Early results using this approach on 2D MR and echocardiographic image sequence data were encouraging [40], but it was appreciated that there were a number of difficulties with the approach, including the lack of including temporal information in the modeling, the difficulties deriving motion from segmented boundaries and perhaps most importantly, the need to address the full 3D motion problem.

This work evolved in several directions. One effort focused on the integration of temporal and full spatiotemporal information/modeling into the motion recovery process [46]. This work, by John McEachen for his thesis, continued to work on 2D image sequence data and took on a decidedly classical Electrical Engineering flavor as the basic strategy was based on recursive filtering incorporating a periodicity constraint. A second effort was centered on integrating several sources of tracking cues into the frame-to-frame LV motion estimation process. The initial idea was aimed at using the boundary-based shape cues described above, along with Eulerian estimates of displacement found by integrating Magnetic Resonance phase ve- 
locity information. The integration of shape with direct, dense measurements of three-dimensional velocity provides a powerful set of constraints on the interpretation of motion from image data. This work was primarily done by Fran cois Meyer, a postdoctoral fellow in IPAG at this time (later a faculty member in the group), who had worked on rigid body motion problems in computer vision during his Ph.D. studies in France. While these data provide direct measures of velocity, they are extremely noisy. A Kalman filtering approach was developed here using spatial and temporal constraints to determine an optimal estimate of the cardiac kinematics [47].

The third research direction that evolved from our initial shape-based 2D cardiac motion tracking work was anchored in two key concepts. The first concept was grounded in the understanding in the cardiology clinical research literature at this time that the quantification of LV function from noninvasive image sequences was found to be more robust if one looked at the relative measurement of LV myocardial wall thickening as opposed to just absolute point displacement (see e.g. [48]). The second concept was that in order to fully understand LV function, one had to appreciate that the heart is a threedimensional, nonrigidly deforming object and that the quantification of this deformation likely had to be derived from the full 4-dimensional ( 3 spatial dimensions plus time) image information. Wall thickening was thus only one component of a more complete measure of myocardial deformation: the local strain tensor.

These same basic ideas were also taking hold at the time in the MR imaging community, driven by the development of MR grid tagging for following tissue deformation [49], [50].

Thus, IPAG efforts on cardiac nonrigid motion in the mid1990's further developed by extending our 2D shape-based motion tracking strategies to three dimensions using 3D curvature to track surface-based motion derived from cine-gradient echo MRI. The earliest efforts were performed by Amir Amini and Jim Duncan, during Amir's postdoctoral studies at Yale [51]. These ideas were further developed in the Ph.D. theses of Pengcheng Shi [52] and then Xenios Papademetris [53]. The curvature matching process represented by equation 5 above was now based on matching the similarity of the principal curvatures between an endocardial or epicardial surface patch at time $i$ to a set of candidate patches within a search region at time $i+1$. A key next step, anchored in Pengcheng Shi's efforts, was the development of an interpolation approach that could accept these image-derived, surface-based displacements and then create quantitative measures of mechanical strain across the entire LV myocardial volume. This work was published at the first CVRMed conference in 1995 [54] and moved IPAG into the area of using more realistic physical models to try to capture the underlying myocardial tissue properties and serve as a basis for integrating information and presenting quantitative measurements of strain. The advantage of this direction was that there was an entire cardiac biomechanics community [55] that had spent decades trying to develop accurate forward models of the LV. The initial IPAG efforts in this area used simple, linear elastic biomechanical models (realized via a stiffness matrix $\mathbf{K})$, assumed infinitesimal strains between any two time frames and assumed that a sparse set of displacements, $\mathbf{U}^{m}$, could be found from 3D shape tracking. The volume between the endocardial and epicardial surfaces at each time frame was meshed and the following Newtonian equation was then solved using a finite element strategy at all points at each time frame to get a dense set of displacements $U$, where the $A$ matrix is used to weight the confidence in the shape matches:

$$
K U=A\left(U-U^{m}\right)
$$

Further extensions were then incorporated to integrate shape based estimates of displacement with velocity estimates measured using phase velocity MR, now in a fully 4D framework and using a continuum mechanical model of the heart [56], [57].

As the physical modeling work continued to mature and evolve in the graduate work of Xenios Papademetris [58], [53], [59], the formulation was viewed from a Bayesian standpoint and the biomechanical model of the heart was augmented to include muscle fiber directions. Quantified 3D measures of strain could now be reliably derived from a moving sequence of segmented myocardial surfaces on a desktop workstation. An example of these derived strains are shown in Figure 12. This methodology was implemented in a software platform that is usable by collaborators and has led to advances in the cardiology literature on regional myocardial deformation [60].

Critical to these efforts was the notion that displacement information could come from a variety of data sources, including shape cues, MR tags, and/or MR phase velocity information. The shape tracking strategy permits the approach to be used across imaging platforms. For example, further work extended these techniques directly to 3D ultrasound data enabling the possibility of such measurements from a low cost and portable modality [61], shown in Figure 13.

These ideas in realistic soft tissue modeling were further developed and applied to the related problem of computing brain deformation during neurosurgery [62], [63]. Biomechanical models are again of extreme value here in order to accurately model the brain and track its deformation incorporating the effects of gravity. This work stems from the thesis of Oskar Škrinjar. First, intraoperative sparse point measurements were used to drive the model [62], [64]. A finite element computational approach was used to implement the biomechanical model. Later, stereo images of the exposed brain surface were used to provide a dense set of measurements for driving the model. Stereo reconstruction methods were derived for this purpose [65] to determine the changing surface of the brain. This process is shown in Figure 14.

Registration Methods: Over the years, a number of us in IPAG have worked on different registration problems with different approaches reflecting both the application area and the underlying methodology, starting with rigid methods and moving to nonrigid. We developed methods for rigid registration incorporating constraints using linear programming [66] as well as methods using genetic methods for improved optimization [67]. Nonrigid methods were developed using elastic and fluid models that were augmented with structural constraints thus combining gray level matching with features [68]. This work, by Yongmei Wang during her graduate work, used these constraints to help to bring the gray level matching into concordance with the underlying anatomy. During his postdoctoral 

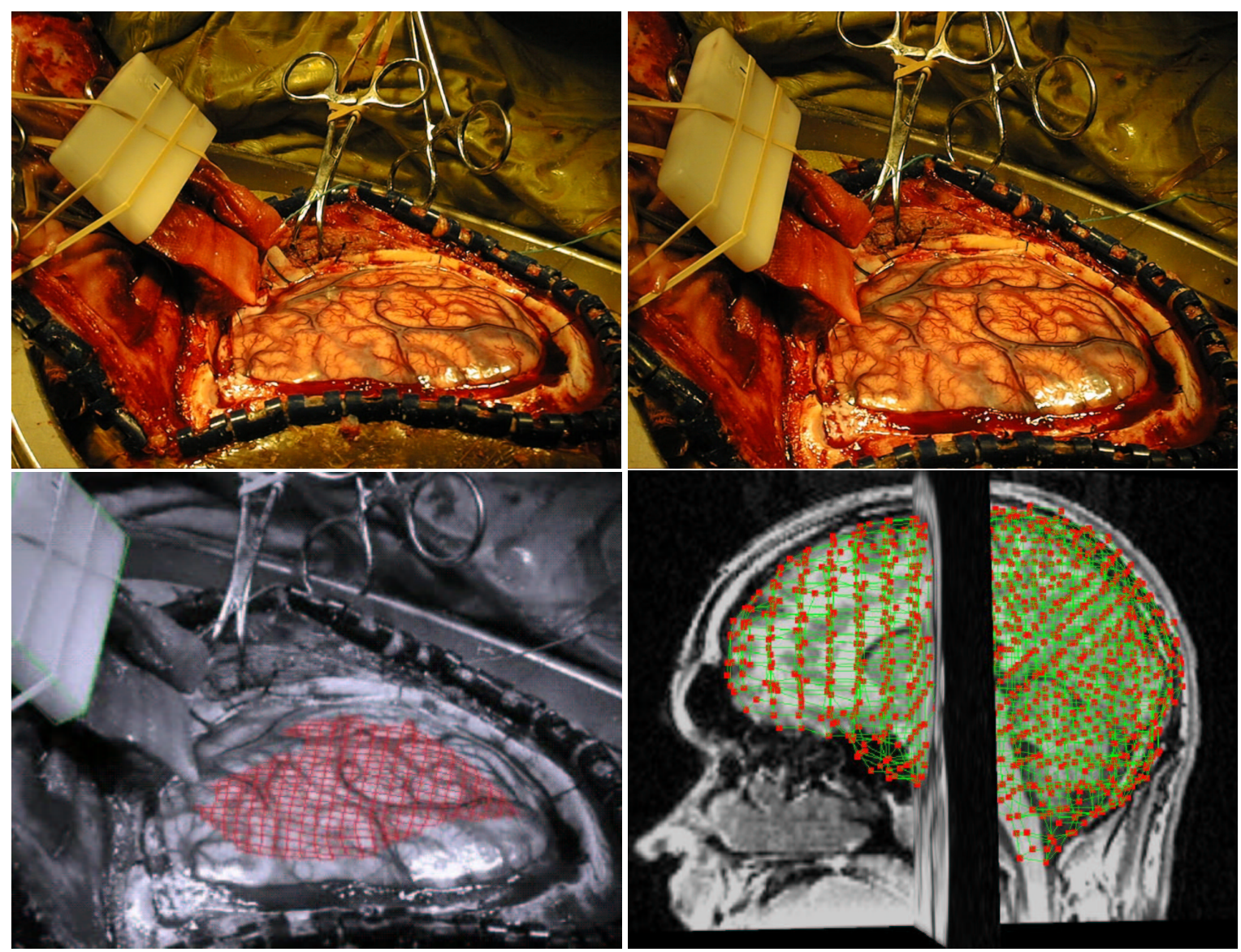

Fig. 14. Intraoperative stereo image pairs (top) can be used to reconstruct the exposed brain surface (bottom left) which can then be used as a constraint in the biomechanical model for determining brain shift (bottom right).

study, Colin Studholme, worked on the problem of magnetic resonance (MR) distortion correction mapping echo planar image data to conventional MR. He developed methods to account for both geometric and intensity distortions within the EPI data by constraining nonrigid mutual information based registration using the physical basis of the distortion [69].

Anand Rangarajan, a faculty member in the group at this period, and his student Haili Chiu, developed the technique robust point matching (RPM) for nonrigid registration [70], [71]. They formulated registration completely in terms of point features and a corresponding match matrix. The match matrix converges to a specification of correspondence, and a corresponding nonrigid transformation, with outlier rejection using the "soft-assign" technique.

Another approach to non-rigid correspondence between closed curves was pursued by Hemant Tagare. Generalizations of monotonic one-to-one correspondences, called bimorphisms, were shown to be a class of "monotonic" curves on the torus, which is a product space of two closed curves. By suitably defining a metric on the torus, the shapes of the two curves can be compared and an optimal correspondence can be found [72], [73]. Figure 15 shows an example. There are several advantages of this idea. The theory clearly lays out the geometry of correspondences. Also, the optimal correspondence is symmetric, i.e. the same answer is obtained irrespective of the order in which the two curves are compared. The last property is impor-

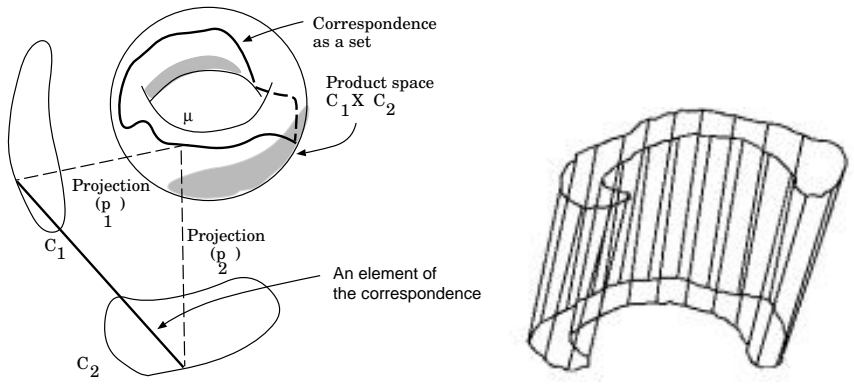

Fig. 15. The correspondence between two closed curves is shown to be a curve on the torus, which is the product space of the curves (left). An optimal correspondence from one corpus callosum to another is shown on the right.

tant in applying non-rigid correspondences to motion analysis.

An information theoretic framework was developed for the specific registration problem in radiation therapy treatment planning where one or more low quality 2D portal x-rays (generated from therapeutic energy $\mathrm{x}$-rays) need to be registered to a 3D CT image [74], [75]. Ravi Bansal, in his thesis work, developed an information theoretic approach for this problem where simultaneous segmentation and registration were carried out in an alternating iterative minimax entropy algorithm.

We have also pursued methods for the determination of surface point correspondence for shape comparison based on curvature features and geodesic interpolation [76], [77]. 


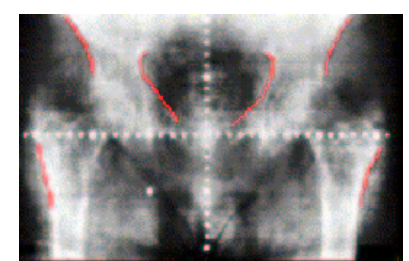

a

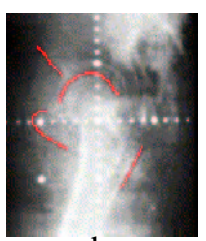

b

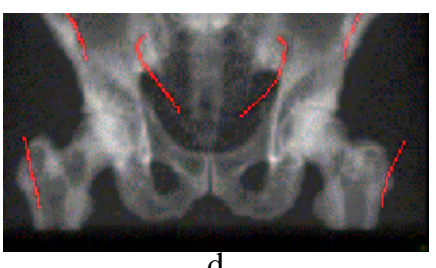

$\mathrm{d}$

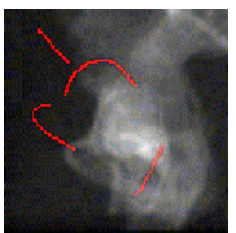

C

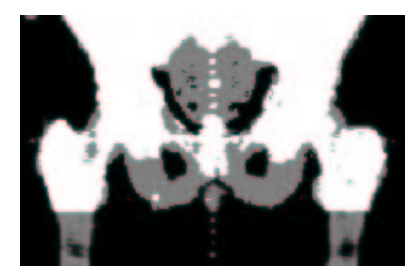

e

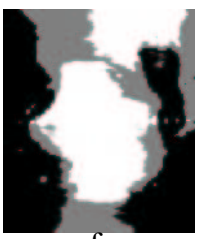

f

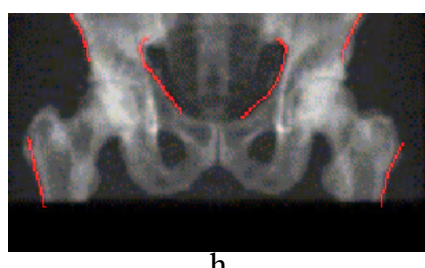

h
Fig. 16. Registration of portal images with digitally reconstructed radiographs (DRR) from CT before (left) and after (right) registration. Manual traces in red indicate degree of correspondence of features. (a) anterior-posterior (AP) portal (b) lateral portal (c) original lateral DRR (d) original AP DRR (e) AP segmentation (f) lateral segmentation $(\mathrm{g})$ registered lateral DRR $(\mathrm{h})$ registered AP DRR.

Measurement: Structure can be characterized in many ways from simple volume measurements to curvature. We have developed a number of techniques for specific structural measurement.

The level set representation is particularly convenient for some structural computations, such as thickness. For any point on the outer cortical surface, the absolute value of $\Psi_{i n}$ at the point is simply the distance from the point to the inner cortical surface. Using this measure, we obtain a thickness map between the inner and outer cortical surfaces, which can be used to study the normal thickness variations in different regions as well as abnormalities, as seen in Figure 17. Cortical surface area is also easily captured and measured from our segmentation approach.

Cortical surface determination facilitates further analysis by the determination of sulcal surfaces [78]. First, sulcal curves at the top and bottom of the sulcus can be automatically traced after the specification of start and end points using dynamic programming based on a surface maximum principal curvature cost function. Sulcal ribbon surfaces (shown in Figure 19) can then be determined between these curves deforming the surface based on the distance function of the surface within the sulcus.

Neuroanatomic measurement applications apply these methods to clinical and scientific tasks such as for structural volume measurement [79], [80]. We have developed methods for shape analysis using factor analysis for the characterization of shape applied to the corpus callosum [81].

Image Databases: Digital medical imaging has made it possible to create and maintain large collections of medical images for research and browsing. Medical image content is

\begin{tabular}{|c||c|}
\hline $\begin{array}{c}\text { Region } \\
\text { (Lobe) }\end{array}$ & $\begin{array}{c}\text { thickness } \\
(\mathrm{mm})( \pm \mathrm{SD})\end{array}$ \\
\hline \hline Left Frontal & $3.40(.43)$ \\
Right Frontal & $3.25(.42)$ \\
Left Posterior* & $3.06(.41)$ \\
Right Posterior* & $3.00(.40)$ \\
\hline
\end{tabular}

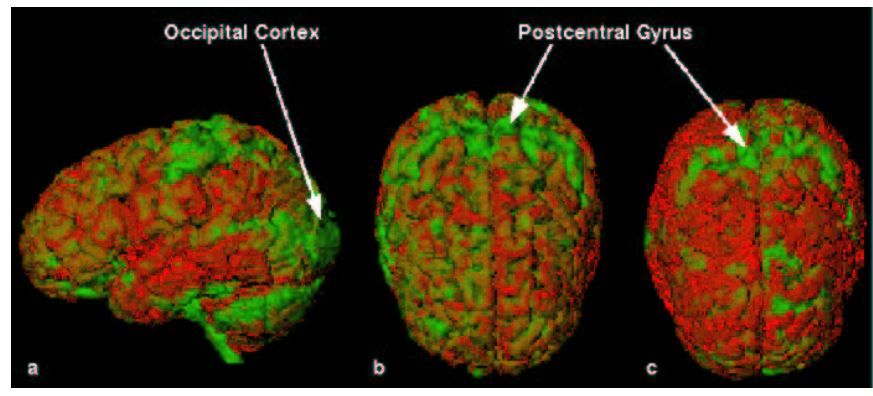

thickness(mm)

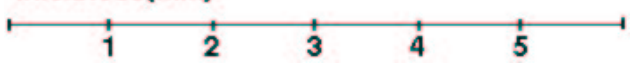

Fig. 17. Measurement of Cortical Thickness. Top: Table reporting mean thickness values for $\mathrm{N}=30$ normal control males. Note: * Posterior region encompasses all parietal, temporal and occipital cortical tissue. Bottom: Thickness plots of 2 normal brains (one brain: a,b; second brain: c) showing marked thinning in the postcentral gyrus and primary and secondary visual cortices in the occipital lobe.

difficult to describe in words and a medical image database is most effective if it exploits image content. Developing the technology for such databases has been a focus of extensive work by Hemant Tagare. An overview of the problems in this area and our overall methodology is given in [82]. Much of our research in this area focuses on indexing strategies for similarity retrieval. Classical indexing strategies assume that features are in a Euclidean space and a metric is available for comparing them. We extend indexing strategies to the non-metric case using interval-valued arithmetic [83] and propose non-Euclidean metric spaces for indexing [84]. Complicated feature comparisons, for example with dynamic programming, can also be indexed [85]. In high dimensional spaces, indexing suffers from the curse of dimension; it becomes increasingly difficult to index uniformly distributed data. Efficient indexing needs to exploit non-uniformity in data distribution and we have developed adaptive indexing strategies for this purpose [86], [87]. These strategies modify the indexing tree so that the resulting tree is guaranteed to be more efficient than the original one.

Other aspects of medical image databases have also been developed. While visiting our group, Sennay Ghebreab developed a tool for creating and editing graphical image database schemas [88]. Some interesting medical image features have also been developed. One example is the notion of arrangements of organs, which describe the manner in which different organs appear embedded in the image [89]. Arrangement is related to the Voronoi diagram of the organs and it is possible to define a metric comparing different arrangements.

Although our group has focused on developing image databases in the context of medical images, a surprising and gratifying application of this technology has been in biology in marine mammal research [90], [91]. 


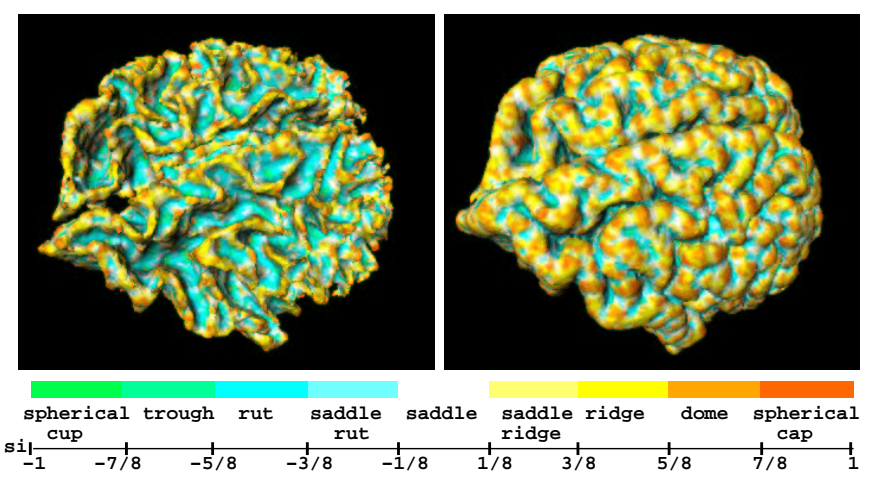

Fig. 18. The inner and outer cortical surfaces of a brain colored according to the corresponding curvature measure [92].

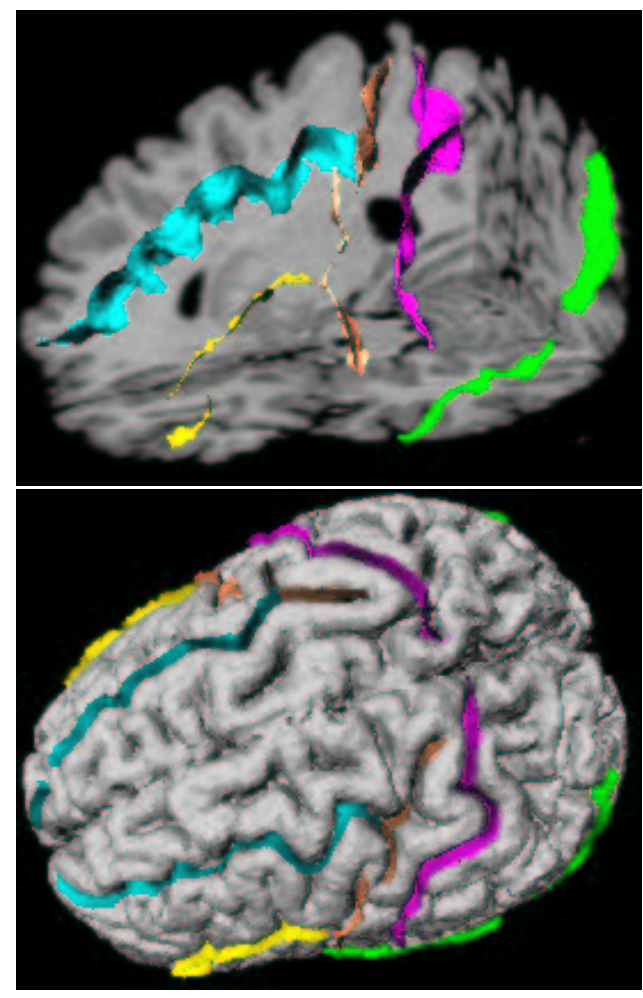

Fig. 19. Sulcal surfaces shown with cut-away view of brain (top) and on outer cortical rendering (bottom).

\section{ReCEnt Developments}

Two developments at Yale have led to the better integration of education and research in medical imaging: the formation of a program in biomedical engineering within the Faculty of Engineering and a section of bioimaging sciences within the Department of Diagnostic Radiology.

In 1996, with the generous support of the Whitaker Foundation, we embarked on the formation of a biomedical engineering program at Yale with medical imaging as a core area (along with biotechnology and biomechanics). This program was designed to combine the strengths of engineering and medicine at Yale and has steadily grown since then. The program provides both undergraduate and graduate students who can participate in research in medical imaging.

In the early Spring of 2001, the Image Processing and Analysis Group was formally united with groups in the areas of MR

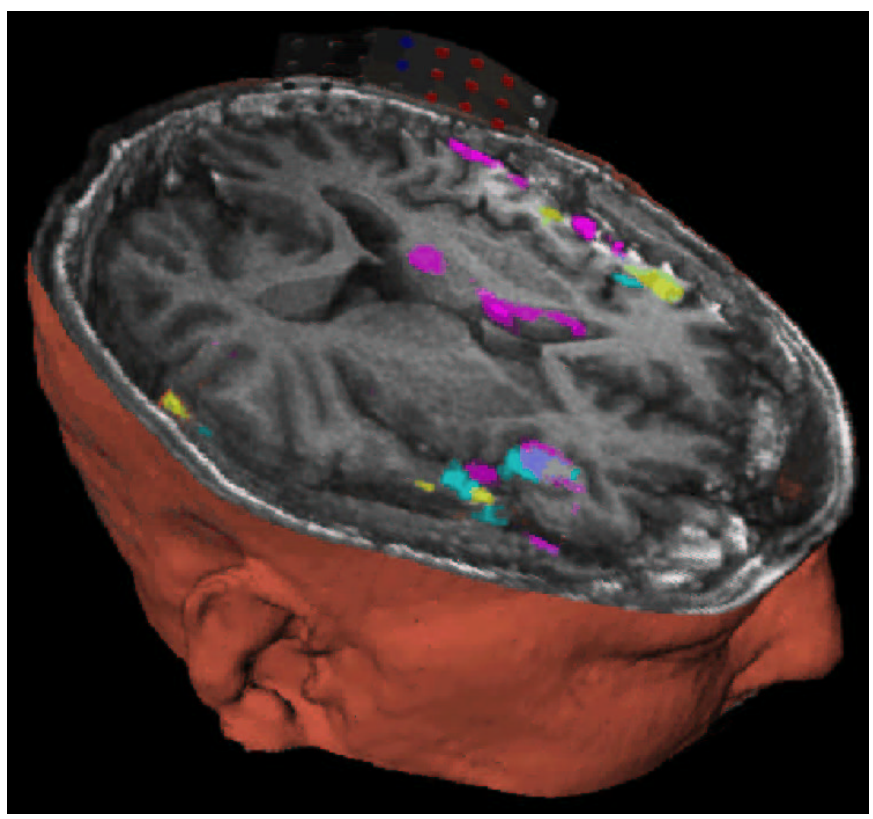

Fig. 20. Bioimaging and Intervention in Neocortical Epilepsy: An example of the integrated display of anatomical and functional magnetic resonance images, along with electrophysiological information taken from a patient. This represents an initial version of the neurosurgical navigational framework being developed on this grant.

Physics, Nuclear Medicine Physics and NMR Spectroscopy within the Department of Diagnostic Radiology. This structure was termed the Section of Bioimaging Sciences and Professor Duncan was named as the director and Professor Doug Rothman, an NMR spectroscopist, named as co-director. This unification of research groups in Diagnostic Radiology has already resulted in a tighter connection between the image formation and image analysis aspects of medical imaging research. In particular, as a direct result of closer collaboration with the other groups in the Section, we were awarded the first new research grant from the National Institute of Bioimaging and Biomedical Engineering for a biomedical engineering research partnership grant entitled "Bioimaging and Intervention in Neocortical Epilepsy" aimed at the study of epilepsy through MR imaging and spectroscopy with analysis techniques culminating in improved image guided neurosurgery for the treatment of epilepsy (see Figure 20).

The ultimate source of the productivity of the group is the talents of the many students and scholars who have worked here. With the recent addition of Xenios Papademetris to the faculty, we currently have four professors (Duncan, Tagare, Staib, Papademetris), two research scientists, four postdocs and 11 graduate students. In addition, George Zubal, has long been a close affiliate to the group, focusing on quantification in nuclear medicine. On the strength of all their efforts, IPAG will continue to be productive.

\section{Future Directions}

Certainly, our core work using organ and tissue-level diagnostic medical images in segmenting and measuring structure and function, image registration, and tracking and quantifying motion and deformation will remain at the center of IPAG's ef- 
forts in the years to come. Continuing to explore how our approaches can be integrated or economized is always something at the forefront of our thinking: e.g. how can image intensity and image-derived feature information be combined to develop more robust segmentation and registration algorithms or how can parameters found at a higher level of abstraction (e.g. strain in an infarcted region of the left ventricle) be used to guide the extraction of useful low level image features?

However, we also expect to be drawn in new and exciting directions based on our exposure to emerging collaborations with our colleagues in MR spectroscopy and physics, different clinical areas related to image-guided intervention and structural and functional imaging at the cellular and molecular level. While we will be able to dovetail some of these efforts with methodological approaches we are already developing, we expect to be drawn to entirely new directions that include: tracking multiple nonrigid moving objects with complex evolving relationships (e.g. cell body motion and tubule growth), estimating statistical mixtures of biochemical information represented at each voxel in a variety of image datasets attempting to probe biologically meaningful information (e.g. MR spectroscopy, molecular imaging using fluorescent and/or radiolabeled probes) and designing close-to-real-time updating strategies regarding tissue and tool movement in interventional procedures.

\section{GuIDING PRINCIPLES}

Within IPAG, we feel fortunate to have been able to create a stable scientific, financial and administrative environment where medical image analysis researchers interested in applying well-grounded mathematical and computational concepts to problems in medical imaging can flourish. There have been several guiding principles that have helped us:

1) Take the time to find and maintain good clinical and technical collaborators who have interesting problems that are pushing the current limits of medical image analysis and processing technology. The profile of a good collaborator can vary, but generally has these traits:

- he/she realizes that you are looking for interesting problems in your own (medical image analysis) research area, related to the methodology and underlying formulation of these issues - and they want to walk side-by-side with you toward common goals.

- is someone who you can genuinely resonate with and get along with.

- is someone who has an appreciation and some understanding of the field of medical image analysis.

We have had longstanding and successful collaborations with a number of scientists at Yale including Robert Schultz (Child Study Center), Albert Sinusas (Cardiology), Dennis Spencer (Neurosurgery) and Todd Constable (Diagnostic Radiology).

2) Go after problems that push the current state-of-the-art; avoid problems that apply established/routine ideas in computer vision to problems in medical image analysis that don't require at least some original thinking. However, keep in mind the requirements of medical image analysis in terms of the need to validate.
3) Maintain a presence, including publications, conference attendance, etc., in both the basic fields of computer vision and image processing as well as the more focused field of medical image analysis. The primary medical image analysis conferences have been IPMI (Information Processing in Medical Imaging) and MICCAI (Medical Image Computing and Computer Assisted Intervention) (and its predecessors). Participation in the clinical/biomedical application communities is also important (e.g. cardiology, neurology, neuroscience, etc).

4) Permit junior faculty to develop in an independent manner, but provide guidance to try to develop a cohesive and complementary group of researchers.

5) Develop a stable internal source of funds from the Department/School that carries over year-to-year.

6) Encourage collaboration and cooperation with other medical image analysis groups. The intellectual life at IPAG has periodically been enriched by visiting students and scholars. We have had particularly good relationships with visitors from Guy's Hospital through Dave Hawkes (Glynn Robinson, Colin Studholme), University of Amsterdam through Arnold Smeulders (Marcel Worring, Sennay Ghebreab), Utrecht University through Max Viergever (Wiro Niessen, Josien Pluim, Rik Stokking) and INRIA-Sophia Antipolis through Nicholas Ayache (Eric Bardinet).

7) Insure access to graduate students either through participation in a medical imaging sciences graduate program or through appointments and teaching with an appropriate department (e.g. EE, BME, CS, Physics, etc.)

\section{ACKNOWLEDGEMENTS}

Special thanks to Hemant Tagare for his help with this document.

James S. Duncan, Guest Editor

Image Processing and Analysis Group

Section of Bioimaging Sciences

Departments of Diagnostic Radiology and

Electrical Engineering

Yale University

New Haven, CT 06520 USA

Lawrence H. Staib, Guest Editor

Image Processing and Analysis Group

Section of Bioimaging Sciences

Departments of Diagnostic Radiology and

Electrical Engineering

Yale University

New Haven, CT 06520 USA

\section{REFERENCES}

[1] D. Gerogiannis and S. C. Orphanoudakis, "Load balancing requirements in parallel implementations of image feature extraction tasks," IEEE Transactions on Parallel \& Distributed Systems, vol. 4, no. 9, pp. 9941013, Sept. 1993. 
[2] K. P. Andriole, J. S. Duncan, S. C. Orphanoudakis, and T. Shibata, "Development of a 3-D discontinuity detector and linking algorithm for tracking vessels in medical images," in Proc. Twelfth Annual Northeast Bioengineering Conf., 1986, pp. 137-140.

[3] G. R. Gindi and A. F. Gmitro, "Optical feature extraction via the radon transform," Optical Engineering, vol. 23, no. 5, pp. 499-506, Sep-Oct 1984.

[4] A. F. Gmitro, V. Tresp, and G. R. Gindi, "Videographic tomography I: Reconstruction with parallel-beam projection data," IEEE Trans. Med. Imaging, vol. 9, no. 4, pp. 366-375, 1990.

[5] M. Lee, A. Rangarajan, I. G. Zubal, and G. Gindi, "A continuation method for emission tomography," IEEE Trans. Nucl. Sci., vol. 40, no. 6, pp. 2049-2058, 1993.

[6] G. Gindi, M. Lee, A. Rangarajan, and I. G. Zubal, "Bayesian reconstruction of functional images using anatomical information as priors," IEEE Trans. Med. Imaging, vol. 12, no. 4, pp. 670-680, 1993.

[7] I. G. Zubal, C. R. Harrell, E. O. Smith, Z. Rattner, G. R. Gindi, and P. B. Hoffer, "Computerized 3-dimensional segmented human anatomy," Med. Phys., vol. 21, no. 2, pp. 299-302, 1994.

[8] Zubal Phantom: http://ipagwww.med.yale.edu/zubal.

[9] G. R. Gindi and J. S. Duncan, "A complete object recognition system as a computer vision course project," IEEE Transactions on Education, vol. 30, no. 3, pp. 142-150, August 1987.

[10] J. S. Duncan, "Knowledge directed left ventricular boundary detection in equilibrium radionuclide angiocardiography," IEEE Trans. Med. Imaging, vol. 6 , no. 4, pp. 325-336, 1987.

[11] J. Utans and G. R. Gindi, "Improving convergence in hierarchical matching networks for object recognition," in Advances in Neural Information Processing 5, S. Hanson, J. Cowan, and L. Giles, Eds. Morgan Kaufmann, San Mateo, CA, 1993.

[12] L. H. Staib, X. Zeng, A. Chakraborty, R. T. Schultz, and J. S. Duncan, "Shape constraints in deformable models," in Handbook of Medical Imaging: Processing and Analysis, I. Bankman, Ed. Academic Press, San Diego, 2000.

[13] A. Chakraborty, L. H. Staib, and J. S. Duncan, "Deformable boundary finding in medical images by integrating gradient and region information," IEEE Trans. Med. Imaging, vol. 15, no. 6, pp. 859-870, 1996.

[14] A. Chakraborty and J. S. Duncan, "Game theoretic integration for image segmentation," IEEE Trans. Pattern Anal. Machine Intell., vol. 21, no. 1, pp. 12-30, 1999.

[15] L. H. Staib, Parametrically Deformable Contour Models for Image Analysis, Ph.D. thesis, Yale University, New Haven, CT, 1990.

[16] L. H. Staib and J. S. Duncan, "Boundary finding with parametrically deformable models," IEEE Trans. Pattern Anal. Machine Intell., vol. 14, no. 11, pp. 1061-1075, 1992.

[17] M. Worring, A. W. M. Smeulders, L. H. Staib, and J. S. Duncan, "Parameterized feasible boundaries in gradient vector fields," Comp. Vision and Image Understanding, vol. 63, no. 1, pp. 135-144, Jan. 1996.

[18] L. H. Staib and J. S. Duncan, "Model-based deformable surface finding for medical images," IEEE Trans. Med. Imaging, vol. 15, no. 5, pp. 720 731, 1996.

[19] L. H. Staib and J. S. Duncan, "Left ventricular analysis using parametrically deformable models," in AAAI Spring Symposium Series: Artificial Intelligence in Medicine, Stanford University, Mar. 1988.

[20] L. H. Staib and J. S. Duncan, "Left ventricular analysis from cardiac images using deformable models," in Proc. Computers in Cardiology, pp. 427-430. IEEE Comp. Soc., Los Alamitos, CA, 1988.

[21] T. Cootes, A. Hill, C. Taylor, and J. Haslam, "The use of active shape models for locating structures in medical images," in Information Proc. Med. Imaging, H. H. Barrett and A. F. Gmitro, Eds., pp. 33-47. LNCS 687, Springer, Berlin, 1993.

[22] Y. Wang and L. H. Staib, "Boundary finding with correspondence using statistical shape models," in Proc. Comp. Vision Pattern Recog., 1998, pp. 338-345.

[23] Y. Wang and L. H. Staib, "Statistical shape and smoothness models for boundary finding with correspondence," IEEE Trans. Pattern Anal. Machine Intell., vol. 22, no. 7, pp. 738-743, 2000.

[24] H. D. Tagare, "Deformable 2-d template matching using orthogonal curves," IEEE Trans. Med. Imaging, vol. 16, no. 1, pp. 108-117, 1997.

[25] Y. Chen, H. D. Tagare, S. Thiruvenkadam, F. Huang, D. Wilson, A. Geiser, K. Gopinath, and R. Briggs, "Using prior shapes in geometric active contours in a variational framework," Int. J. Computer Vision, vol. 50, no. 3, pp. 315-328, 2002.

[26] T. Ma, Active Contour Models: Consistency, Stability, and Parameter Estimation, Ph.D. thesis, Yale University, 1997.

[27] T. Ma and H. D. Tagare, "Bias and stability of active contours with euclidean and non-euclidean arc-lengths," IEEE Trans. Image Proc., vol. 8, no. 11, pp. 1549-1559, 1999.
[28] L. H. Staib, A. Chakraborty, and J. S. Duncan, "An integrated approach for locating neuroanatomical structure from MRI," Int. J. Patt. Recog. Art. Intell., vol. 11, no. 8, pp. 1247-1269, 1997, (Special Issue on MR Brain Image Analysis).

[29] G. Owen, Game Theory, Academic Press, 1982.

[30] T. Basar and G. J. Olsder, Dynamic noncooperative game theory, Academic Press, 1982.

[31] H. I. Bozma and J. S. Duncan, "A game-theoretic approach to integration of modules," IEEE Trans. Pattern Anal. Machine Intell., vol. 16, pp. 1074-1086, 1994.

[32] J. Nash, "Equilibrium points in n-person games," Proc. Natl. Acad. Sci. USA, vol. 36, pp. 48-49, July 1950.

[33] P. Baxandall and H. Liebeck, Vector Calculus, Oxford University Press, 1986.

[34] X. Zeng, L. H. Staib, R. T. Schultz, and J. S. Duncan, "Volumetric layer segmentation using coupled surfaces propagation," in Computer Vision and Pattern Recognition, 1998, pp. 708-715.

[35] X. Zeng, L. H. Staib, R. T. Schultz, and J. S. Duncan, "Segmentation and measurement of the cortex from 3D MR images," in Medical Image Computing and Computer-Assisted Intervention (LNCS 1496), Berlin, 1998, pp. 519-530, Springer.

[36] X. Zeng, L. H. Staib, R. T. Schultz, and J. S. Duncan, "Segmentation and measurement of the cortex from 3D MR images using coupled surfaces propagation,” IEEE Trans. Med. Imaging, vol. 18, no. 10, 1999.

[37] D. W. Shattuck and R. M. Leahy, "Brainsuite: An automated cortical surface identification tool," in Medical Image Computing and ComputerAssisted Intervention (MICCAI), S. L. Delp, A. M. DiGoia, and B. Jaramaz, Eds., Berlin, 2000, vol. Lecture Notes in Computer Science, Vol. 1935, pp. 51-61, Springer-Verlag.

[38] X. Papademetris and J. S. Duncan, "Cardiac image analysis: Motion and deformation," in Handbook of Medical Imaging, Volume 2: Medical Image Processing and Analysis, M. Sonka and J. M. Fitzpatrick, Eds. SPIE Press, 2000

[39] G. E. Mailloux, A. Bleau, M. Bertrand, and R. Petitclerc, "Computer analysis of heart motion from two-dimensional echocardiograms," IEEE Trans. Biomed. Eng., vol. 34, pp. 356-364, 1987.

[40] J. S. Duncan, R. L. Owen, L. H. Staib, and P. Anandan, "Measurement of non-rigid motion in images using contour shape descriptors," in Proc. Comp. Vision Pattern Recog., June 1991, pp. 318-324.

[41] D. Metaxas and D. Terzopoulos, "Constrained deformable superquadrics and nonrigid motion tracking," in Computer Vision and Pattern Recognition, G. Medioni and B. Horn, Eds. 1991, pp. 337-343, IEEE Press.

[42] A. Pentland and S. Sclaroff, "Closed-form solutions for physically based shape modeling and recognition," IEEE Trans. Pattern Anal. Machine Intell., vol. 13, no. 7, pp. 715-729, July 1991.

[43] J. Park, D. Metaxas, and L. Axel, "Analysis of left ventricular wall motion based on volumetric deformable models and MRI-SPAMM," Medical Image Analysis, vol. 1, no. 1, pp. 53-71, 1996.

[44] S. N. Gupta and J. L. Prince, "On variable brightness optical flow for tagged MRI," in Information Processing in Medical Imaging, June 1995, pp. 323-334.

[45] N. Pelc, M. Drangova, L. Pelc, Y. Zhu, D. Noll, B. Bowman, and R. Herfkens, "Tracking of cyclic motion with phase-contrast cine mr velocity data," Journal of Magnetic Resonance Imaging, vol. 5, pp. 339-345, 1995.

[46] J. McEachen and J.S. Duncan, "Shape-based tracking of left ventricular wall motion," IEEE Trans. Med. Imaging, vol. 16, no. 3, pp. 270-283, June 1997.

[47] F. Meyer, R. T. Constable, A. Sinusas, and J. Duncan, "Tracking myocardial deformation using phase contrast velocity fields: A stochastic approach," IEEE Transactions on Medical Imaging, vol. 15, no. 4, pp. 453-465, 1996.

[48] F. H. Sheehan, E. L. Bolson, H. T. Dodge, D. G. Mathey, J. Schofer, and H. W. Woo, "Advantages and applications of the centerline method for characterizing regional ventricular function," Circulation, vol. 74, pp. 293-305, 1986.

[49] E. Zerhouni, D. Parish, W. Rogers, A. Yang, and E. Shapiro, "Human heart tagging with MR imaging-a method for noninvasive assessment of myocardial motion," Radiology, vol. 169, pp. 59-63, 1988.

[50] L. Axel and L. Dougherty, "MR imaging of motion with spatial modulation of magnetization," Radiology, vol. 171, pp. 841-845, 1989.

[51] A. Amini, R. L. Owen, P. Anandan, and J. S. Duncan, "Non-rigid motion models for tracking the left ventricular wall," in Information Processing in Medical Imaging, Alan C. F. Colchester and David J. Hawkes, Eds. 1991, vol. 511 of Lecture Notes in Computer Science, pp. 343-357, Springer.

[52] P. Shi, Image Analysis of 3D Cardiac Motion Using Physical and Geometrical Models, Ph.D. thesis, Yale University, May 1996. 
[53] X. Papademetris, Estimation of 3D Left Ventricular Deformation from Medical Images Using Biomechanical Models., Ph.D. thesis, Yale University, May 2000.

[54] P. Shi, G. Robinson, A. Chakraborty, L. Staib, R. Constable, A. Sinusas, and J. Duncan, "A unified framework to assess myocardial function from 4D images," in Comp. Vision, Virtual Reality and Robotics in Med. (CVRMed '95, LNCS 905), N. Ayache, Ed., Berlin, 1995, pp. 327-340, Springer.

[55] P. J. Hunter, A. McCulloch, and P. Nielsen, Eds., Theory of Heart, Springer-Verlag, Berlin, 1991.

[56] P. Shi, A.J. Sinusas, R.T. Constable, and J.S. Duncan, "Volumetric deformation analysis using mechanics-based data fusion: Applications in cardiac motion recovery," Int. J. Computer Vision, vol. 35, no. 1, pp. 87-107, Nov. 1999.

[57] P. Shi, A. Sinusas, R. Constable, E. Ritman, and J. Duncan, "Pointtracked quantitative analysis of left ventricular motion from 3D image sequences," IEEE Trans. Med. Imaging, vol. 19, no. 1, pp. 36-50, 2000.

[58] X. Papademetris, P. Shi, D. P. Dione, A. J. Sinusas, and J. S. Duncan, "Recovery of soft tissue object deformation using biomechanical models," in Information Proc. Med. Imaging, M. Šámal A. Kuba and A. ToddPokropek, Eds., Berlin, 1999, pp. 352-357, Springer-Verlag.

[59] X. Papademetris, A. J. Sinusas, D. P. Dione, R. T. Constable, and J. S Duncan, "Estimation of 3D left ventricular deformation from medical images using biomechanical models," IEEE Trans. Med. Imaging, vol. 21, no. 7, pp. 786-800, July 2002.

[60] A. J. Sinusas, X. Papademetris, R. T. Constable, D. P. Dione, M. D. Slade, P. Shi, and J. S. Duncan., "Quantification of 3-d regional myocardial deformation: Shape-based analysis of magnetic resonance images," American Journal of Physiology: Heart and Circulatory Physiology, vol. 281, pp. H698-H714, August 2001.

[61] X. Papademetris, A. J. Sinusas, D. P. Dione, and J. S. Duncan, "Estimation of 3D left ventricular deformation from echocardiography," Med. Image Anal., vol. 5, no. 1, pp. 17-28, 2001.

[62] O. Škrinjar, D. Spencer, and J. Duncan, "Brain shift modeling for use in neurosurgery," in Medical Image Computing and Computer-Assisted Intervention (LNCS 1496), W. M. Wells, A. Colchester, and S. Delp, Eds., Berlin, 1998, pp. 641-649, Springer.

[63] O. Škrinjar, A. Nabavi, and J. S. Duncan, "Model-driven brain shift compensation," Medical Image Analysis, vol. 6, no. 4, pp. 361-373, December 2002.

[64] O. Škrinjar and J. Duncan, "Real time 3D brain shift compensation," in Information Proc. Med. Imaging, M. Šámal A. Kuba and A. ToddPokropek, Eds., Berlin, 1999, pp. 42-55, LNCS 1613, Springer.

[65] O. Škrinjar, H. Tagare, and J.S. Duncan, "Surface growing from stereo images," in Proc. Comp. Vision Pattern Recog., June 2000, pp. II:571576.

[66] G. Zubal, H. Tagare, L. Zhang, and J. Duncan, "3D registration of intermodality medical images," in IEEE Conf. Eng. Med. Biology, 1991, pp. 293-294.

[67] L. H. Staib and X. Lei, "Intermodality 3D medical image registration with global search," in Proc. IEEE Workshop Biomedical Image Anal., 1994, pp. 225-234.

[68] Y. Wang and L. H. Staib, "Physical model based non-rigid registration incorporating statistical shape information," Med. Image Anal., vol. 4 pp. 7-20, 2000.

[69] C. Studholme, R.T. Constable, and J.S. Duncan, "Non-rigid spin echo MRI registration incorporating an image distortion model: Application to accurate alignment of fMRI to conventional MRI," IEEE Trans. Med. Imaging, vol. 19, no. 11, pp. 1115-1127, November 2000.

[70] A. Rangarajan, H. Chui, E. Mjolsness, S. Pappu, L. Davachi, P. GoldmanRakic, and J. Duncan, "A robust point-matching algorithm for autoradiograph alignment," Med. Image Anal., pp. 379-398, 1997.

[71] H. Chui, J. Rambo, J. S. Duncan, R. T. Schultz, and A. Rangarajan, "Registration of cortical anatomical structures via robust 3D point matching," in Information Proc. Med. Imaging, A. Kuba, M. Šámal, and A. ToddPokropek, Eds., Berlin, 1999, pp. 168-181, LNCS 1613, Springer.

[72] H. Tagare, "Shape-based nonrigid correspondence with applications to heart motion analysis," IEEE Trans. Med. Imaging, vol. 18, no. 7, pp. 570-579, 1999.

[73] H. D. Tagare, D. O'Shea, and D. Groisser, "Shape based non-rigid correspondence for plane curves," J. Math. Imaging Vision, vol. 16, pp. 57-68, 2002.

[74] R. Bansal, L. Staib, Z. Chen, A. Rangarajan, J. Knisely, R. Nath, and J. Duncan, "A minimax entropy registration framework for patient setup verification in radiotherapy," Computer Aided Surgery, vol. 4, no. 6, pp. 287-304, 1999.

[75] R. Bansal, L. Staib, Z. Chen, A. Rangarajan, J. Knisely, R. Nath, and J. Duncan, "Entropy-based, dual-portal-to-3DCT registration incorporat- ing pixel correlation," IEEE Trans. Med. Imaging, vol. 22, no. 1, pp. 29-49, 2003.

[76] Y. Wang, B. S. Peterson, and L. H. Staib, "Shape-based 3D surface correspondence using geodesics and local geometry," in Proc. Comp. Vision Pattern Recog., 2000, vol. II, pp. 644-651.

[77] Y. Wang, B. S. Peterson, and L. H. Staib, "3D brain surface matching based on geodesics and local geometry," Comp. Vision and Image Understanding, vol. 89, no. 2-3, pp. 252-271, Jan. 2003.

[78] X. Zeng, L. H. Staib, R. T. Schultz, H. Tagare, L. Win, and J. S. Duncan, "A new approach to 3D sulcal ribbon finding from MR images," in Medical Image Computing and Computer-Assisted Intervention (LNCS 1679), Berlin, 1999, pp. 148-157, Springer.

[79] R. T. Schultz, N. K. Cho, L. H. Staib, L. E. Kier, J. M. Fletcher, S. E Shaywitz, D. P. Shankweiler, J. C. Gore, J. S. Duncan, and B. A. Shaywitz, "Brain morphology in normal and dyslexic children: The influence of sex and age," Ann. Neurol., vol. 35, no. 6, pp. 732-742, June 1994.

[80] B. S. Peterson, B. Vohr, L. H. Staib, C. Cannistraci, A. Dolberg, K. A. Schneider, K. H. Katz, M. Westerveld, S. Sparrow, A. Anderson, C. Duncan, R. W. Makuch, J. C. Gore, and L. R. Ment, "Regional brain volume abnormalities are associated with long-term cognitive outcome in preterm infants," J. Am. Med. Assoc., vol. 284, pp. 1939-1947, 2000.

[81] B. S. Peterson, P. A. Feineigle, L. H. Staib, and J. C. Gore, "Automated measurement of latent morphological features in the human corpus callosum," Human Brain Mapping, vol. 12, no. 4, pp. 232-245, 2001.

[82] H. D. Tagare, C. C. Jaffe, and J. Duncan, "Medical image databases: A content-based retrieval approach," J. Am. Med. Informatics Assoc., vol. 4, no. 3, pp. 184-198, 1997.

[83] H. D. Tagare, "Efficient retrieval without a metric," in IEEE Visual '97, San Diego, 1997.

[84] H. D. Tagare, "Efficient retrieval in image databases," in SPIE Conf., San Diego, July 2000.

[85] G. Robinson, H. Tagare, J. Duncan, and C. Jaffe, "Medical image collection indexing: Shape-based retrieval using kd trees," Computerized Medical Imaging and Graphics, vol. 20, no. 4, pp. 209-217, 1996.

[86] H. D. Tagare, "Increasing retrieval efficiency by index tree adaptation," in IEEE Workshop on Content-based Access of Image and Video Libraries, 1997.

[87] X. Qian and H. D. Tagare, "Optimally adapted indexing trees for medical image databases," in ISBI, Washington D.C., July 2002.

[88] S. Ghebreab, M. Worring, H. D. Tagare, and C. C. Jaffe, "SCHEMed: a visual tool for definition and data entry in medical image databases," in IEEE Visual'97, San Diego, 1997.

[89] H. Tagare, F. Vos, C. C. Jaffe, and J. S. Duncan, "Arrangement: A spatial relation between parts for evaluating similarity of tomographic section," IEEE Trans. Pattern Anal. Machine Intell., vol. 17, no. 9, pp. 880-893, 1995.

[90] G. R. Hillman, H. D. Tagare, K. Elder, A. Drobyshevski, D. Weller, and B. Würsig, "Shape descriptors computed from photographs of dolphin dorsal fins for use as database indices," in Proc. IEEE Eng. Medicine Biology Society, Nov. 1998.

[91] G. R. Hillman, B. Würsig, N. Kehtarnavaz, D. W. Weller, A. Kreho, A. Drobyshevski, K. Elder, B. N. Araabi, T. McKinney, G. Gailey, and H. D. Tagare, "Individual identification of dolphins in field photographs: a computer-based system," in The Society for Marine Mammalogy, 13th Biennial Conference on the Biology of Marine Mammals, 1999.

[92] J. J. Koenderink and A. J. van Doorn, "Surface shape and curvature scales," Image and Vision Computing, vol. 10, no. 8, pp. 557-565, Oct. 1992. 